\section{Concomitant presentation of myocarditis and non-bacterial thrombotic endocarditis in a patient with granulomato- sis with polyangiitis}

\author{
Amirhossein Yazdi, ${ }^{1}$ Mehrnaz Olfat, ${ }^{1}$ \\ Hassan Riahi Beni, ${ }^{2}$ Behnood Bikdeli ${ }^{3}$ \\ 'Department of Cardiology, School \\ of Medicine, Hamedan University of \\ Medical Sciences, Hamedan, Iran; \\ 2Department of Cardiology, School \\ of Medicine, University of Medical \\ Sciences, Tehran, Iran; \\ ${ }^{3}$ Center for Outcomes Research and
}

Evaluation, and Department of Internal

Medicine, Yale-New Haven Hospital, New

Haven, CT, USA

\section{Abstract}

Granulomatosis with polyangiitis (GPA) is a necrotizing vasculitis. The etiology of the disease is not completely known. In this report, we describe a 47-year-old male who presented with ongoing dyspnea. He is a known case of GPA for 3 years based on skin biopsy and positive antineutrophil cytoplasmic antibody. The electrocardiogram showed changes suggestive for myocarditis. A transthoracic and trans esophageal echocardiogram revealed left ventricular systolic dysfunction, thickened left atrial and ventricular wall and vegetation on mitral valve. Blood cultures were negative. Myocarditis and nonbacterial thrombotic endocarditis (NBTE) were diagnosed. As far as we know, NBTE is a rare manifestation of GPA and its presentation with myocarditis is not reported to date. This case indicates the uncommon manifestations of GPA and notifies the importance of performing echocardiography in order to prevent life threatening cardiac complications of the disease.

\section{Introduction}

Granulomatosis with polyangiitis (GPA) is a necrotizing vasculitis of small vessels that affect respiratory tract and the kidneys. ${ }^{1}$ The etiology of the disease is not completely explained yet, but it seems that both environmental and genetic factors play roles in this disease. ${ }^{2}$ GPA involve many organs; among them kidneys, ear, nose, throat and lungs are more frequently involved; but eyes, cartilages, skin and other organs can also be affected. ${ }^{1}$ In a patient presenting with clinical findings sug- gestive for GPA, the confirm diagnosis is made by a tissue biopsy or sufficient positive titer of antineutrophil cytoplasmic antibody (c-ANCA) which is highly specific for GPA.,4 Suggested treatment for GPA consists of IV glucocorticoids and oral cyclophosphamide. ${ }^{5,6}$ About 50$70 \%$ of patients experience relapsing episodes. ${ }^{1}$ According to different studies, echocardiographic abnormalities due to cardiac involvement in GPA is relatively common, including systolic dysfunction, pericardial effusion, valvular involvement and so far. 7,8 Nonbacterial thrombotic endocarditis (NBTE) is a rare manifestation of GPA and to our knowledge its concomitant presentation with myocarditis is not reported to date. Herein, we report a case of GPA, who diagnosed with both myocarditis and NBTE.

\section{Case Report}

This patient was a 47-year-old male, who was diagnosed with GPA 3 years earlier with recurrent episodes of polyarthritis, petechiae and abnormal renal function test which lead to end stage renal disease (ESRD). The diagnosis of GPA was made based on skin biopsy and cANCA titer of 31.5 (greater than 9.0 is considered as positive). He was started on immunosuppressive therapy (prednisolone and azathioprine), but he has discontinued treatment after few months. Now, He does dialysis 2 times a week. After months of being relatively healthy, he presented to our outpatient clinic with increased shortness of breath and orthopnea, which limited his daily activities. On physical examination, he was alert and afebrile, diminished breath sounds at both sides specially left side and moist rahle up to half of both lungs. He was admitted to our hospital for more evaluation.

Laboratory data, including complete blood count (CBC), blood urea nitrogen (BUN), creatinine (Cr), serum electrolytes and erythrocyte sedimentation rate (ESR) are shown in Table 1.

Chest X-ray showed small bilateral pleural effusion also perm catheter for dialysis (Figure 1).

The ECG showed: sinus tachycardia, T wave inversion, LVH voltage criteria (Figure 2).

Transthoracic echocardiography (TTE) was done and it revealed LV systolic dysfunction and a small and irregular vegetation on anterior mitral valve leaflet (Figure 3 ).

Transesopheageal echocardiography was also performed (TEE) and it confirmed previous findings.

Several blood cultures were negative and no growth was observed after five days of incubation. No sign or symptom compatible with cancer was mentioned. No embolic event was occurred. We requested serological testing
Correspondence: Amirhossein Yazdi, Ekbatan Hospital, Hamedan 6515737461, Iran.

Tel.: +98.912 .3098490 - Fax: +98.811 .8268034$

E-mail: a.h.yazdi89@gmail.com

Key words: granulomatosis with polyangiitis, myocarditis, nonbacterial thrombotic endocarditis, antineutrophil cytoplasmic antibody.

Contributions: AY, designing, supervising and editing the manuscript; MO, gathering data, writing the manuscript; HRB, BB, technical support, conceptual advice and editing the manuscript.

Conflict of interests: the authors declare no potential conflict of interests.

Received for publication: 20 September 2013.

Revision received: 19 January 2014.

Accepted for publication: 24 March 2014.

This work is licensed under a Creative Commons Attribution NonCommercial 3.0 License (CC BYNC 3.0).

CC Copyright A. Yazdi et al., 2014

Licensee PAGEPress, Italy

Rheumatology Reports 2014; 6:5078

doi:10.4081/rr.2014.5078

(including Anti cadiolipinAb, lupus anticoagulant test, ANA, Anti ds-DNA, p-ANCA, C3, C4 and $\mathrm{CH} 50$ ). All were within normal limits, except: c-ANCA: 20 (greater than 9.0 is considered as positive)

The patient underwent emission computed tomography (99m Tc-MIBISPECT) scanning (Figure 4) after 10 days of immunosuppressive therapy $(60 \mathrm{mg} / \mathrm{d}$ prednisolone and $100 \mathrm{mg} / \mathrm{d}$ azathioprine). It showed persistent hypo activity in apical antroseptal segment and reversible defects in the mid to apical portion of anterior wall, hypo activity of septum, LV dilation and dramatically increased global LV function to $50 \%$.

The diagnosis was myocarditis and non bacterial thrombotic endocarditis (NBTE) due to relapse of the disease.

According to insignificant mitral regurgitation and no obvious embolic event patient was discharged with increased dose of immunosuppressive drugs, $80 \mathrm{mg}$ ASA and conventional heart failure management. He was strongly recommended to a complete course of treatment, close symptoms and echocardiography follow up.

\section{Discussion}

In echocardiography, mobile masses on valve constructions usually are vegetations 
from subacute bacterial endocarditis, tumors, or (rarely) thrombi. In the absence of symptoms, benign tumors such as fibroelastomas often are considered. The finding of a large, intracardiac mass caused by NBTE, without known malignancy or systemic lupus erythematous or anti phospholipid antibody syndrome, is rare.

Vegetations produced by NBTE are found in $\approx 1.2 \%$ of autopsies, but the reported prevalence varies widely ( $0.3 \%$ to $9.3 \%)$ and predominantly involves patients with coexisting malignancy, sepsis, burns, or disseminated intravascular coagulation. ${ }^{9}$

However, autoimmune disease are found in

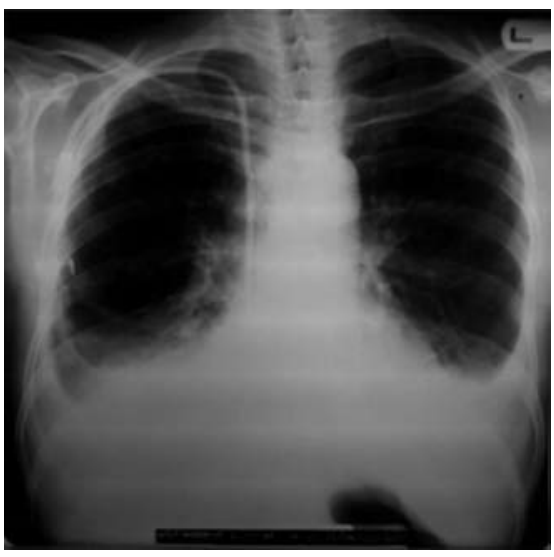

Figure 1. Chest X-ray shows bilateral pleural effusion and perm catheter for dialysis.

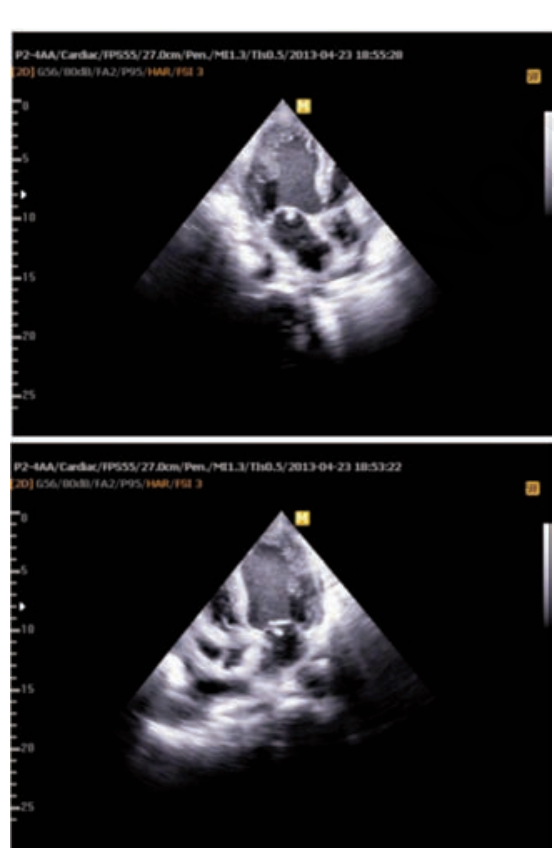

Figure 3. Transthorasicechocardiograohy shows left ventricle systolic dysfunction and a vegetation on anterior mitral valve leaflet.
Table 1. Complete blood count, blood urea nitrogen, creatinine and erythrocyte sedimentation rate.

\begin{tabular}{lcc} 
Tests & Result & Units \\
Red blood cell & 3.66 & $\mathrm{~mL} / \mathrm{cumm}$ \\
Hemoglobin & 8.5 & $\mathrm{~g} / \mathrm{d}$ \\
\hline Hematocrit & 29 & $\%$ \\
Platelet & 113 & $\mathrm{cumm}$ \\
\hline Mean corpuscular hemoglobin concentration & 29 & $\mathrm{~g} / \mathrm{dL}$ \\
Mean corpuscular hemoglobin & 23.2 & $\mathrm{pg}$ \\
\hline Blood urea nitrogen & 60 & $\mathrm{mg} / \mathrm{dL}$ \\
Creatinine & 12.5 & $\mathrm{mg} / \mathrm{dL}$ \\
\hline Erythrocyte sedimentation rate & 40 & $\mathrm{~mm}$
\end{tabular}

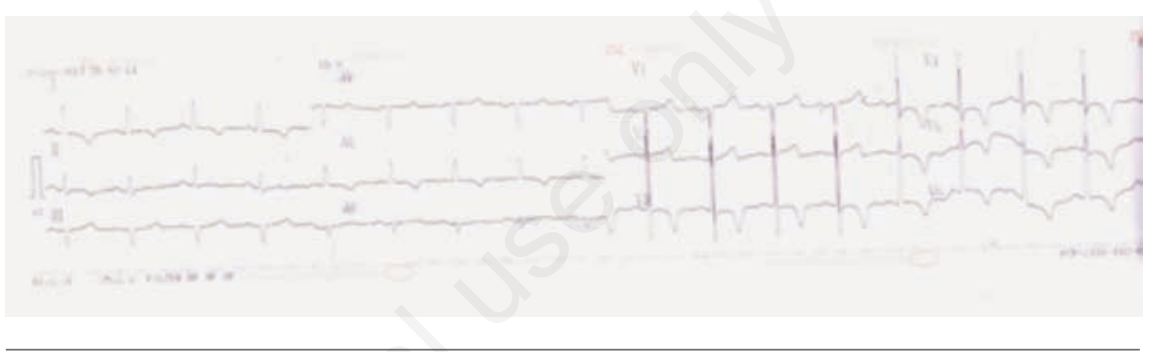

Figure 2. Electrocardiogram shows tachycardia, T wave inversion and LVH voltage criteria.

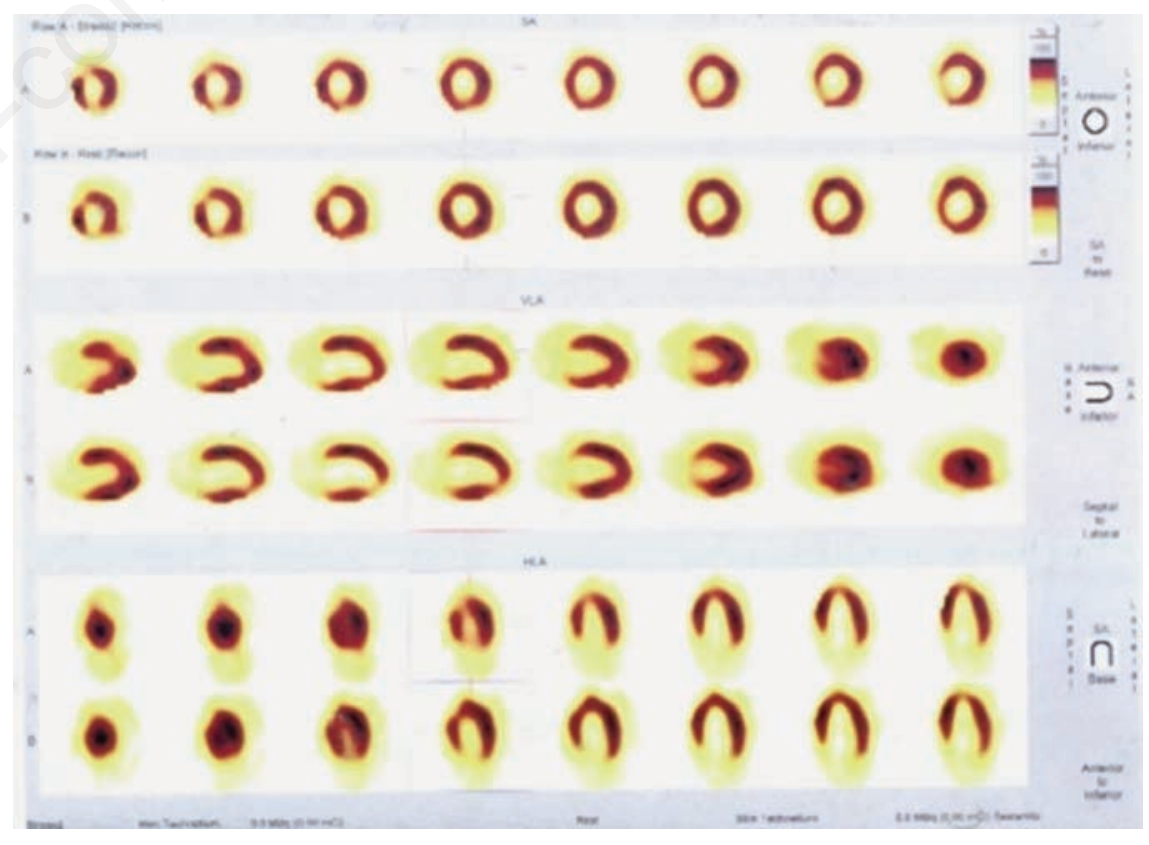

Figure 4. Computed tomography scanning shows findings compatible with myocarditis. 
$60 \%$ of patients who undergo surgical resection for an intracardiac mass caused by NBTE.10 Among authoimmune disease, antiphospholipid syndrome (APS) and systemic lopus erythematouse (SLE) are more frequently associated with this condition. ${ }^{11}$ NBTE in patients with GPA is rarely reported in literatures and in some cases has been presented by embolic events. ${ }^{12,13}$ To the best of our knowledge, this was the first case of granulomatosis with polyangiitis with both NBTE and myocarditis, which was diagnosed before life threatening embolic events.

\section{Conclusions}

Herein, we reported an unusual patient who presented with dyspnea. The ECG changes suspected us to myocarditis. We performed TTE which showed LV systolic dysfunction and a mass on the anterior mitral valve leaflet, suggestive for NBTE. Our diagnosis was confirmed with TEE and computed tomography scanning. Our case emphasizes the need for searching for cardiac involvement in all autoimmune and connective tissue diseases by echocardiography to prevent life threading complications.

\section{References}

1. Langford CA, Fauci AS. The vasculitis syndromes. In: Jameson J, Longo D, Kasper D, et al., eds. Harrison's principles of internal medicine. 18th ed. New York: McGraw-Hill; 2011. pp 2789-9272.

2. Mahr AD, Neogi T, Merkel PA. Epidemiology of Wegener's granulomatosis: lessons from descriptive studies and analyses of genetic and environmental risk determinants. Clin Exp Rheumatol 2006;24:S82-91.

3. Venning MC, Quinn A, Broomhead V, Bird AG. Antibodies directed against neutrophils (C-ANCA and P-ANCA) are of distinct diagnostic value in systemic vasculitis. Q J Med 1990;77:1287-96.

4. Göbel U, Briedigkeit L, Voigt B, et al. [Wegener's granulomatosis and anti-cytoplasm antibodies]. Z Gesamte Inn Med 1990;45:638-43. [Article in German].

5. Fauci AS, Haynes BF, Katz P, Wolff SM. Wegener's granulomatosis: prospective clinical and therapeutic experience with 85 patients for 21 years. Ann Intern Med 1983;98:76-85.

6. Hoffman GS, Kerr GS, Leavitt RY, et al. Wegener granulomatosis: an analysis of 158 patients. Ann Intern Med 1992;116: 488-98.
7. Oliveira GH, Seward JB, Tsang TS, Specks U. Echocardiographic findings in patients with Wegener granulomatosis. Mayo Clin Proc 2005;80:1435-40.

8. Morelli S, Gurgo Di Castelmenardo AM, et al. Cardiac involvement in patients with Wegener's granulomatosis. Rheumatol Int 2000;19:209-12.

9. Lopez JA, Ross RS, Fishbein MC, Siegel RJ. Nonbacterial thrombotic endocarditis: a review. Am Heart J 1987;113:773-84.

10. Eiken PW1, Edwards WD, Tazelaar HD, et al. Surgical pathology of nonbacterial thrombotic endocarditis in 30 patients, 1985-2000. Mayo Clin Proc 2001;76:120412.

11. Demin AA, Chapaeva NN, Trifonova MA. [Pseudoinfectious endocarditis]. Klin Med (Mosk) 2008;86:13-7. [Article in Russian].

12. Paik ML, MacLennan GT, Seftel AD. Embolic testicular infarction secondary to nonbacterial thrombotic endocarditis in Wegener's granulomatosis. J Urol 1999; 161:919-20.

13. Jiménez Caballero PE, Segura Martín T. Cardioembolic stroke secondary to nonbacterial endocarditis in Wegener disease. Eur J Neurol 2007;14:683-5. 